\title{
Exponential decay of spin-spin correlation between distant defect states produced by contour hydrogenation of polycyclic aromatic hydrocarbon molecules
}

\author{
G. Chiappe and E. Louis \\ Departamento de Física Aplicada, Unidad Asociada del CSIC and Instituto Universitario de Materiales, \\ Universidad de Alicante, San Vicente del Raspeig, 03690 Alicante, Spain \\ A. Guijarro \\ Departamento de Química Orgánica and Instituto Universitario de Síntesis Orgánica, \\ Universidad de Alicante, San Vicente del Raspeig, 03690 Alicante, Spain \\ E. San-Fabián \\ Departamento de Química Física, Unidad Asociada del CSIC and Instituto Universitario de Materiales, \\ Universidad de Alicante, San Vicente del Raspeig, 03690 Alicante, Spain \\ J. A. Vergés* \\ Departamento de Teoría y Simulación de Materiales, Instituto de Ciencia de Materiales de Madrid (CSIC), \\ Cantoblanco, 28049 Madrid, Spain \\ (Received 5 November 2012; published 18 March 2013)
}

\begin{abstract}
The first few low-lying spin states of alternant polycyclic aromatic hydrocarbon (PAH) molecules of several shapes showing defect states induced by contour hydrogenation have been studied both by $a b$ initio methods and by a precise numerical solution of Pariser-Parr-Pople (PPP) interacting model. In accordance with Lieb's theorem, the ground state shows a spin multiplicity equal to one for balanced molecules, and it gets larger values for imbalanced molecules (that is, when the number of $\pi$ electrons on both subsets is not equal). Furthermore, we find a systematic decrease of the singlet-triplet splitting as a function of the distance between defects, regardless of whether the ground state is singlet or triplet. For example, a splitting smaller than $0.001 \mathrm{eV}$ is obtained for a medium size $\mathrm{C}_{46} \mathrm{H}_{28} \mathrm{PAH}$ molecule (di-hydrogenated [11]phenacene) showing a singlet ground state. We conclude that $\pi$ electrons unbound by lattice defects tend to remain localized and unpaired even when long-range Coulomb interaction is taken into account. Therefore they show a biradical character (polyradical character for more than two defects) and should be studied as two or more local doublets. The implications for electron transport are potentially important since these unpaired electrons can trap traveling electrons or simply flip their spin at a very small energy cost.
\end{abstract}

DOI: 10.1103/PhysRevB.87.125126

PACS number(s): 31.10.+z, 31.15.aq, 33.15.Kr, 75.50.Xx

\section{INTRODUCTION}

There has been intense activity in the search for magnetism in graphene during the last years. Point defects, flake geometry, adatoms, etc., have been invoked as possible origins of magnetism. Useful references throughout a vast literature can be a seminal theoretical work, ${ }^{1}$ a mature review by Yazyev, ${ }^{2}$ and a recent state of the art experimental work. ${ }^{3}$ In any case, as said in the last reference, experimental evidence for antiferromagnetism remains both scarce and controversial.

Our starting point is a theoretical method we have developed a few years ago that explains how a polycyclic aromatic hydrocarbon $(\mathrm{PAH})$ molecule can have a ground state with large spin multiplicity. ${ }^{4}$ It is based on a selective hydrogenation of the molecule contour in such a way that only $\pi$ electrons on one of the two sublattices of the bipartite structure become deactivated. The idea implies to extend the validity of Lieb's theorem, ${ }^{5,6}$ which should apply if the interaction between $\pi$ electrons of PAH's is described by a Hubbard model, to more realistic models and calculations as found in standard quantum chemistry methods based, e.g., in density functional theory. Subsequent computational work has confirmed this idea from a numerical point of view. ${ }^{7,8}$ Actually, we have shown that the long-standing Pariser-Parr-Pople (PPP) model $^{9,10}$ with improved parameters works very well describing low-energy excitations of PAH's. ${ }^{8,11}$ The present experimental capabilities of edge engineering of graphene nanoribbons have been described by Zhang et al. ${ }^{12}$

In this work, we undertake a modest but relevant objective in the quest for magnetism in simple hydrocarbons (including nanographenes). We try to establish the actual stability of triplets and larger spin degeneracies of molecules or clusters that are designed to show a magnetic ground state. Only when the singlet-triplet splitting is large enough compared with $k_{B} T$ the possibility for long-range magnetic correlations among defects can be established. On the other hand, when the spins of electrons localized on different defects produce nearly spin degenerated global states, one has to speak of biradicals for two centers and polyradicals in a more general situation involving many point defects. ${ }^{13}$ In these cases, every radical center should act nearly independently of each other and the best way to understand their properties is as a set of independent local doublets.

If this characterization of defects survives in graphene, the consequences for electronic transport can be tremendous. While a spin correlated set of defects could stay stable if subject to a flux of external electrons, isolated defects could flip their 
spins with minimum energy cost giving rise to an important scattering mechanism.

The rest of the paper is organized as follows. Section II is devoted to give some details of the methods and procedures used in this work. Both standard quantum chemistry methods valid in multiconfigurational cases, and a recently developed Lanczos solution for the interacting PPP model are briefly described. Section III gives our main results together with some discussion. Results are classed together using geometrical characteristics of molecules. First, results for small molecules that allow exact solution of models, second, large di-hydrogenated $[n]$ phenacenes that can be thought as cross sections of typical graphene ribbons with passivated surfaces, and finally, larger molecules that give a preliminary image of what could happen in graphene flakes. The work ends with a few final remarks and conclusions (see Sec. IV).

\section{METHODS AND NUMERICAL PROCEDURES}

The positions of $\mathrm{C}$ atoms in an alternant PAH define clusters than can be thought as small parts of a graphene bipartite lattice. It is well known that when the number of atoms belonging to each sublattice does not coincide, a ground state showing spin multiplicity equal to the number difference plus one is expected, i.e., $\left|N_{A}-N_{B}\right|+1$, where $N_{A}$ and $N_{B}$ are the numbers of atoms in each sublattice. This result has been proven by Lieb $^{5}$ for a Hubbard model on the lattice but its validity extends to real molecules because the PPP model with both local and long-range Coulomb interactions provides a quantitative description of $\pi$ electrons of PAH's. Both models show important differences ${ }^{14}$ but share an important on-site electronic repulsion producing the antiferromagnetic correlations that are at the heart of the theorem. There are many ways to achieve sublattice imbalance, the cluster shape or vacancies addition being among the most popular. Better than removing $\mathrm{C}$ atoms from the molecule, which gives rise to an important atomic relaxation, we prefer the use of extra hydrogenation of selected $\mathrm{C}$ atoms at the molecule contour. In this way, these $\mathrm{C}$ atoms become fourfold coordinated and their corresponding $\pi$ electrons disappear from the relevant band. We have used this method to show how to get an heptuplet as ground state of coronene after passivation of six external $\mathrm{C}$ atoms. ${ }^{4}$

In this work, we study the relative robustness of the predicted ground states. Therefore, lowest excited and ground states are computed on an equal footing. Although results for molecules of several shapes will be presented, the main effort has been devoted to simple bands formed by assembling hexagons in the same way as they appear in phenanthrene and picene among others. These molecules constitute unit cells of ribbons ended in a zigzag structure, a geometry that has been characterized as a possible origin of magnetism in graphene structures. ${ }^{1}$ The addition of one $\mathrm{H}$ atom to each of the molecule ends gives rise to two local imbalanced sublattices, independently of the total numbers of atoms in each sublattice that can be equal or not. We will show that Lieb's ground-state prediction is scrupulously fulfilled but also singlet-triplet splitting systematically reduces as a function of the separation between defects.
We have employed basically two approaches in our numerical work. One is using US GAMESS, ${ }^{15,16}$ that is, a complete quantum chemistry package that allows precise multireference calculations that can be eventually followed by second-order perturbation theory. ${ }^{17,18}$ The second one does not treat the whole electron system since it is restricted to the description of the set of $\pi$ electrons. They are accurately described by a PPP model with a set of recently adjusted parameters. ${ }^{9,10}$ It provides a good description not only for neutral molecules but also for ions and spin excited states. ${ }^{8,11}$ Exact solutions are available for molecules involving 14 or less sites. For larger systems, we rely on a newly developed Lanczos procedure that follows standard methods of quantum chemistry to include electron-electron correlation taking advantage of a preliminary self-consistent field calculation (SCF). The mean-field result is improved using a configuration interaction scheme within a wisely chosen subspace. ${ }^{19}$ Further technical details regarding both quantum chemistry computation and the numerical solution of the many-body PPP model are given in the next sections.

\section{A. Ab initio calculations}

Once the modified PAH molecule is defined, a simple Hückel approach can be used to get a first approximation of the molecular orbitals. Particularly important are those defining the last occupied orbital (HOMO) and the first unoccupied one (LUMO). ${ }^{20}$ By design, our molecules always have two or more states close to zero. These states are exactly degenerated for imbalanced cases and even for certain balanced ones (the chains shown in Fig. 1). Moreover, these orbitals corresponding to unpaired orbitals tend to be more or less localized in the proximity of the sites where $H$ has been added. A definite characterization of these orbitals, however, needs precise many-body calculations. It becomes clear that high-spin states can be obtained when electrons of parallel spin occupy these orbitals. On the other hand, one has several ways to form a singlet. Two electrons of opposite spin can occupy any of the two or more states close to zero, or one up and one down electron can be accommodated in several ways using different orbitals from the bunch of orbitals that are almost degenerated. That means four or more possible

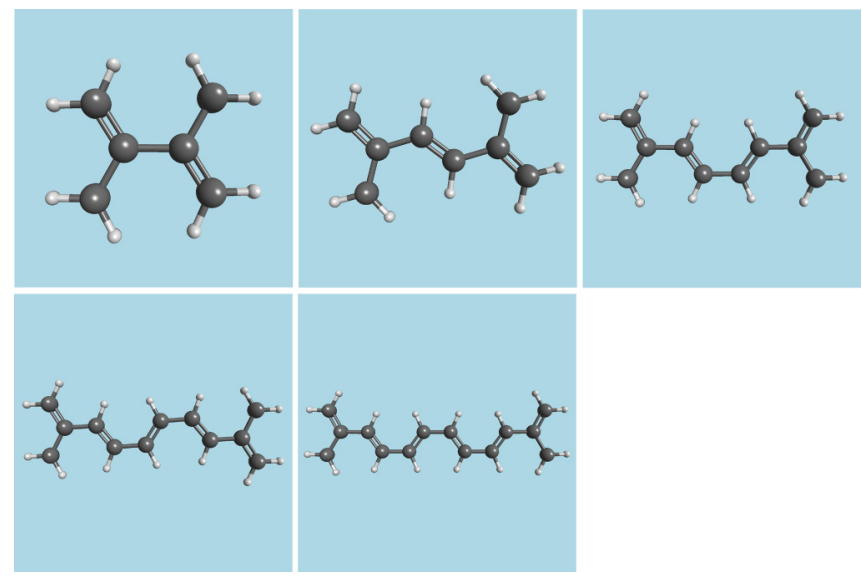

FIG. 1. (Color online) Alternant balanced linear molecules with localized states at the ends. Geometry has been optimized within HF approximation using an STO-6G basis. 
references to start a mean-field calculation, or in other words, a multireference scheme. Therefore, we usually start doing a multiconfiguration self-consistent field (MCSCF) in which all orbitals close to zero are defined as active, that is, their occupation is allowed to get a value between 0 and 2. Later, configuration interaction (CI) is expanded to cover a larger number of molecular orbitals. Many of the results that we shall show below have been obtained for twelve active orbitals describing twelve $\pi$ electrons.

A physically appealing small Slater-type orbital (STO) basis in which orbitals are composed by six Gaussian functions has been systematically used. ${ }^{21}$ Sometimes, more precise cc-pVDZ and PC2 bases ${ }^{22,23}$ have been used in order to test particular results, but for large systems only computations based on a small basis are feasible. As a way to estimate the missing dynamical correlation, second-order perturbation theory has almost always followed MCSCF calculations. Even in a couple of cases, we have tried coupled cluster formalism. Unfortunately, we could not do a multireference calculation: RHF was the uncorrelated starting point for the singlet, and ROHF the mean field for the triplet. In spite of this limitations, a $\operatorname{CCSD}(\mathrm{T})$ calculation allowed us to recover better multireference singlet-triplet splittings.

We have always used MACMOLPLT graphics package by B.M Bode and M.S. Gordon to represent both clusters and 3D wave functions or charge densities. ${ }^{24}$ It is specifically able to read any GAMESS input or output files. In particular, we have used the implemented building capabilities to modify or enlarge many of the molecules studied in this work.

\section{B. Model Hamiltonian}

The PPP model Hamiltonian has been chosen as the standard baseline to describe electronic $\pi-\pi$ correlations. ${ }^{9,10}$ It is simple but realistic enough to reproduce some $a b$ initio results. We use parameters recently obtained for similar molecules. ${ }^{11}$ Strictly speaking, edge $\mathrm{C}$ atoms deactivated by two $\mathrm{H}$ atoms add a new orbital of $\pi$ symmetry to the system (the antibonding combination of $\mathrm{H} s$ orbitals). Nevertheless, this orbital strongly couples to the $\mathrm{C} p z$ orbital forming bonding (occupied) and antibonding (empty) states that are far away from the relevant $\pi$ band. Consequently, our interaction model ignores $\mathrm{C}$ sites that are fourfold coordinated. This approximation reduces the total number of sites, which is an advantage for the numerical solution.

The PPP Hamiltonian contains a noninteracting part $\hat{H}_{0}$ and a term that incorporates the electron-electron interactions $\hat{H}_{I}$ :

$$
\hat{H}=\hat{H}_{0}+\hat{H}_{I}
$$

The noninteracting term is written as

$$
\hat{H}_{0}=\epsilon_{0} \sum_{i=1, N ; \sigma} \hat{c}_{i \sigma}^{\dagger} \hat{c}_{i \sigma}+\sum_{\langle i j\rangle ; \sigma} t_{i j} \hat{c}_{i \sigma}^{\dagger} \hat{c}_{j \sigma}
$$

where the operator $\hat{c}_{i \sigma}^{\dagger}$ creates an electron at site $i$ with spin $\sigma, \epsilon_{0}$ is the energy of carbon $\pi$ orbital, $N$ is the number of unsaturated $\mathrm{C}$ atoms, and $t_{i j}$ is the hopping between nearestneighbor pairs $\langle i j\rangle$ (kinetic energy). Hopping is scaled by a power law: $:^{25}$

$$
t_{i j}=\left(\frac{d_{0}}{d_{i j}}\right)^{3} t_{0}
$$

where $d_{i j}$ is the $\mathrm{C}_{i}-\mathrm{C}_{j}$ distance and $d_{0}=1.41 \AA$. The interacting part is given by

$$
\hat{H}_{I}=U \sum_{i=1, N} \hat{n}_{i \uparrow} \hat{n}_{i \downarrow}+\frac{1}{2} \sum_{i, j=1, N ; i \neq j} V_{i j}\left(\hat{n}_{i}-1\right)\left(\hat{n}_{j}-1\right),
$$

where $U$ is the on-site Coulomb repulsion and $V_{i j}$ is the $\mathrm{C}_{i}-\mathrm{C}_{j}$ intersite Coulomb repulsion given by

$$
V_{i j}=U\left[1+\left(\frac{U}{e^{2} / d_{i j}}\right)^{2}\right]^{-1 / 2}
$$

according to Ohno interpolating formula. ${ }^{26}$ The electronic density operator for spin $\sigma$ at site $i$ is

$$
\hat{n}_{i \sigma}=\hat{c}_{i \sigma}^{\dagger} \hat{c}_{i \sigma},
$$

whereas the operator giving the total electron density at the same site is

$$
\hat{n}_{i}=\hat{n}_{i \uparrow}+\hat{n}_{i \downarrow} .
$$

We use $\epsilon_{0}=-7.61 \mathrm{eV}, t_{0}=-2.34 \mathrm{eV}$, and $U=8.29 \mathrm{eV}^{11}$

The model Hamiltonian is solved using a Lanczos method that follows quantum chemistry standards. A mean-field approach is used to obtain uncorrelated HF molecular orbitals. Afterwards, the interaction model is rewritten in this new basis and CI employed. The crucial point is that the selection of configurations is not based on the occupation of the first wave function but generated from it by successive applications of the many-body Hamiltonian (keep in mind that the use of the Hamiltonian operator to increase the Hilbert space dimension constitutes the essence of the Lanczos method). New configurations are kept only when their participation in the ground state is relevant, that is, when its amplitude is above some carefully chosen threshold. If the threshold is too small, the number of configurations exceeds computational capabilities. On the other hand, if the threshold is too large only a few configurations are generated and correlation is poorly included. Typically, no more than several hundreds of thousands of configurations are used to describe the correlated system. However, the way they have been selected produces a final result showing very good quality. Although our method is applied to a model Hamiltonian and a purely numerical algorithm is used for the selection of configurations, we believe that it shares some similarities with a well documented set of wise criteria of CI selection used for $a b$ initio calculations. ${ }^{27}$ Further details on the whole Lanczos procedure can be found in a recent paper. ${ }^{19}$

\section{RESULTS AND DISCUSSION}

Results are presented classified by the size of the molecules. The first group shows results for chains of increasing length that are not yet aromatic hydrocarbons, but simple organic chains (see Fig. 1). They are included in this work because exact numerical results available for them add strong support 


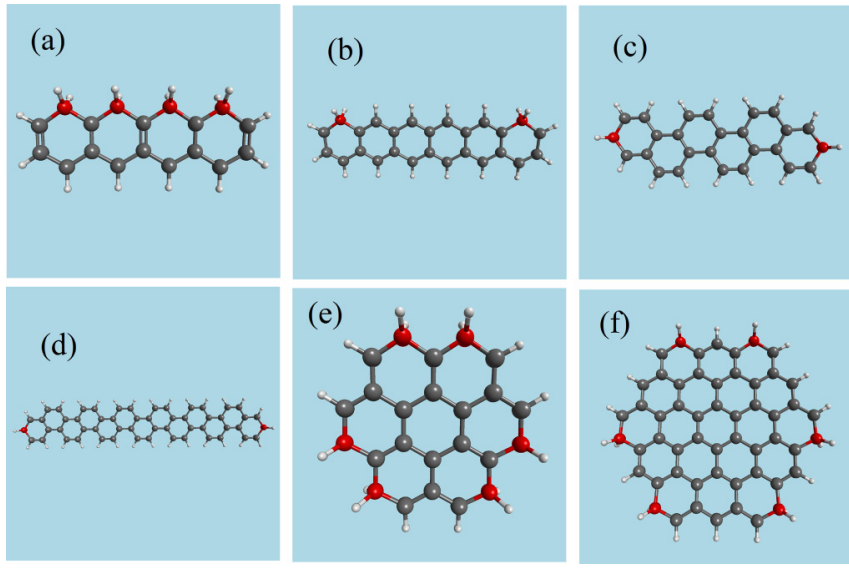

FIG. 2. (Color online) Some of the modified PAH molecules covered by our study. The color of the saturated $\mathrm{C}$ atoms has been changed to red to make clear the place where the localized states studied in this work appear. (a) Tetra-hydrogenated naphthacene, (b) di-hydrogenated hexacene, (c) a relatively short di-hydrogenated [6]phenacene, (d) the longest di-hydrogenated $[n]$ phenacene that we have considered ( $n=13$ ), (e) hexa-hydrogenated coronene and, finally, (f) hexa-hydrogenated supercoronene (a nonstandard name used because it is obtained from coronene by the addition of a layer of hexagonal rings to its perimeter).

to subsequent conclusions. Two examples of defects in a zigzag line follow, namely, tetra-hydrogenated naphthacene and dihydrogenated hexacene shown in Figs. 2(a) and 2(b). Next, the main group of results follows, the one corresponding to large di-hydrogenated $[n]$ phenacenes, i.e., elongated PAH's showing a phenanthrene-like arrangement of hexagonal rings [two examples are shown in Figs. 2(c) and 2(d)]. In this case, both $a b$ initio and Lanczos results for PPP model have been obtained. We end by showing that defect states induced on more regular shapes like coronene and supercoronene can also be described as radical centers that interact weakly as soon as the separation between defects increases [these molecules are given in Figs. 2(e) and 2(f)].

\section{A. Small molecules}

We begin this subsection studying some 1, $\omega$-bis(2propenyl)olygoacethylenes. These molecules are artificial but allow exact results for model calculations and show at a small scale the main characteristics of the systems that constitute our main goal. They have been represented in Fig. 1 and are planar and linear except at the edges. Localized states appear at the molecule extremes just by symmetry. It is clear within one electron theory that the antibonding combination of $\pi$ orbitals of the $\mathrm{CH}_{2}$ groups do not couple to the $\pi$ orbitals on the central $\mathrm{C}$ atoms. Actually, the solution of the corresponding Hückel model gives two states at 0 for any central part with an even number of $\mathrm{C}$ atoms. The occurrence of zero-energy states in the tight-binding Hamiltonian for small balanced pieces of graphene was successfully used by Wang et $a l^{28}$ to propose ultrafast spin logic devices. Their number can be accounted for by a theorem on hexagonal graphs. ${ }^{29}$ In any case, although a triplet ground state could be naively predicted, the full consideration of many-body
TABLE I. Total energies of the chain $\mathrm{C}_{14} \mathrm{H}_{16}$ obtained using different numerical methods. Both PPP model and $a b$ initio results are tabulated.

\begin{tabular}{lccl}
\hline \hline $\mathrm{C}_{14} \mathrm{H}_{16}$ & $S(S+1)$ & PPP model $(\mathrm{eV})$ & ab initio $(\mathrm{eV})$ \\
\hline $\mathrm{RHF}$ & 0 & -137.10 & -14631.38 \\
$\mathrm{ROHF}$ & 2 & -135.44 & -14636.83 \\
$\mathrm{UHF}$ & 3.22 & -140.03 & -14640.28 \\
$\mathrm{CI}$ & 0 & -140.5280 & -14645.1725 \\
$\mathrm{CI}$ & 2 & -140.5278 & -14645.1691 \\
\hline \hline
\end{tabular}

interaction shows that the ground state is a singlet (according with Lieb's theorem for balanced clusters). Next, we are most interested in the actual stability of the ground state, that is, in the value of the singlet-triplet separation. The results discussed in the next paragraph show that the spin multiplicity of the ground state is finally not so decisive because singlet-triplet splitting exponentially decreases as a function of the molecule length.

After a brief geometry optimization at an STO ROHF level, we use the $\mathrm{C}$ positions to define a PPP model that can be exactly solved (fourteen sites is our limit for exact Lanczos results). We took advantage of the fact that all correlation between $\pi$ electrons can also be obtained in this case within an $a b$ initio computation to compare total energies for all approximations that have been used in this work. As shown by the fourth column of Table I, ROHF is significantly better than RHF, that puts two electrons in the bonding combination of distant defect states, but still far from UHF, which occupies defect localized states with electrons of opposite spin in an antiferromagnetic background. Moreover, full CI between $\pi$ orbitals allows electrons to gain about five extra eV. On the other hand, the UHF model results are much closer to the exact energy (third column). Results for shorter chains follow the same trend. Figure 3 gives our results for the singlet-triplet separation of the whole set of chains in a semilogarithmic scale. Results for a Hubbard model trivially derived from the PPP one simply ignoring long-range Coulomb interactions show the same trend. Certainly, the ground state is a singlet (spin multiplicity equal to one) but the first excited state (a triplet, spin multiplicity equal to three) becomes very close as soon as localized states separate. Therefore the splitting becomes negligible for large chains. If the singlet-triplet splitting had to be clearly above $k_{B} T$, one could have spoken of a strong correlation between electrons at the chain ends. However, for negligible splitting, the right image shows two unpaired electrons that barely know about the existence of the other. Since unpaired electrons imply increased reactivity, large chains should be understood as biradicals showing doublets at the molecule extremes. Moreover, since the spin of the electrons does not couple antiferromagnetically to give a singlet, we assume that each of them should behave paramagnetically. ${ }^{3}$

In conclusion, exact results prove that although Lieb's theorem applies to the system predicting the correct ground state, the existence of a merging triplet excited state destroys spin coherence and leads to a molecule that should show large reactivity through the appearance of two independent radical 


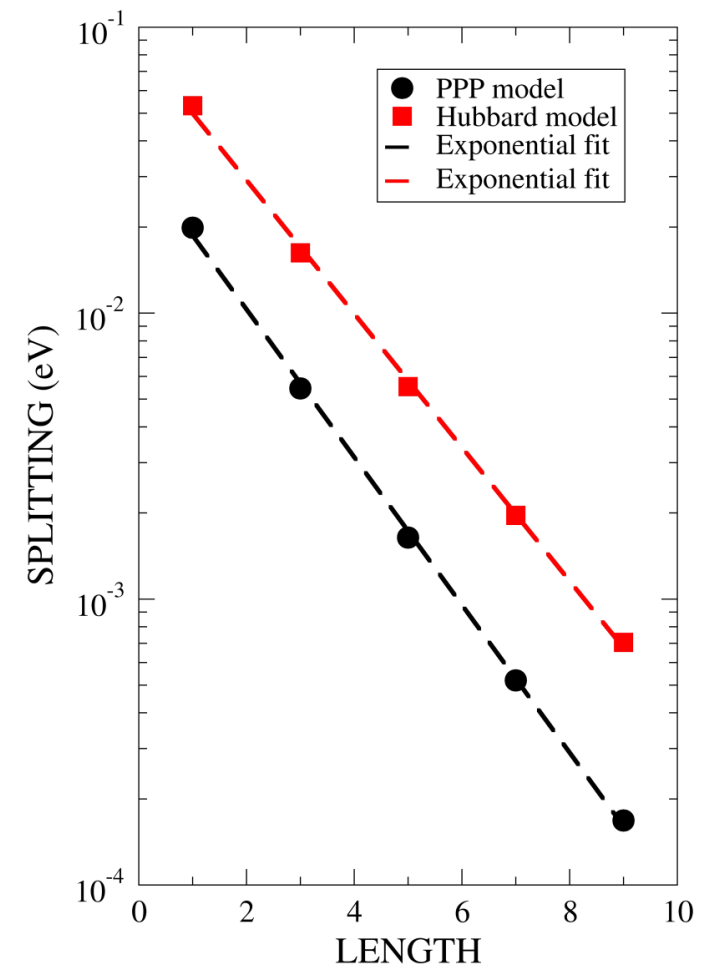

FIG. 3. (Color online) Energy difference between triplet (excited state) and singlet (ground state) of the molecules in Fig. 1 according to exact solutions of PPP and Hubbard models. For the last one, we have used PPP parameters and ignored long-range Coulomb interaction. Exponential decay as a function of defect separation is evident.

centers. ${ }^{13}$ Later, we will show that the same physics appears for more realistic systems although calculations cannot be so precise for larger systems.

Next, we present results for a hydrogenated derivative of naphthacene, which is interesting because its ground state shows spin multiplicity equal to five. Figure 4 shows how four $\mathrm{H}$ atoms have additionally saturated the four $\mathrm{C}$ atoms of one of the molecule sides. Consequently, four lattice sites are deactivated and the molecules ends with an imbalance of four. It exemplifies the behavior of a zigzag border at a small size scale. Both very precise $a b$ initio and exact model results have been gathered for this molecule. They are given in Table II and show a satisfactory consistence. The quintuplet ground state is stable by some tenths of $\mathrm{eV}$, which is more than enough even at room temperature. Therefore we speak of a good spin entanglement for this molecule. The result is not really surprising since defect states are close together and interact. Figure 4 shows the predicted spin density in a ROHF

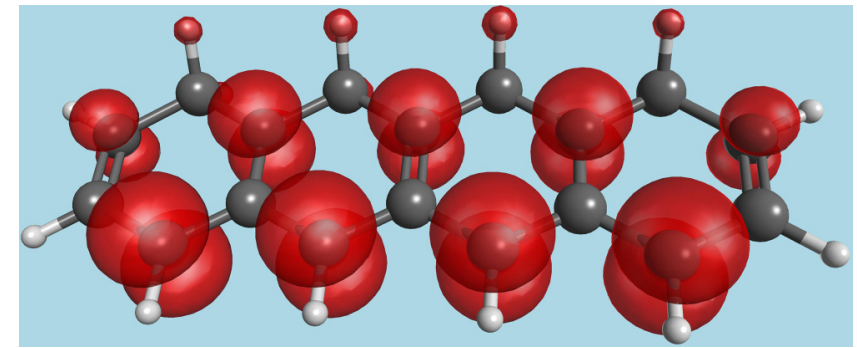

FIG. 4. (Color online) Tetra-hydrogenated naphthacene representation. Total spin density is given for the ROHF approximation just to show how it resides on only one sublattice.

approximation. Unfortunately, GAMESS does not provide the spin density of correlated wave functions.

Although the last result raises expectations for the existence of stable molecules of large spin multiplicity, we show now how di-hydrogenated hexacene behaves as a biradical even if it is predicted to be a triplet. It is formed like tetrahydrogenated naphthacene but with only two separated defects bringing imbalanced sublattices. The molecule is shown in Fig. 5 and several $a b$ initio results are compiled in Table III. Our preferred method, MCSCF followed by second-order perturbation energy, provides consistent results between the small STO basis and a more accurate cc-pVDZ basis. An excited singlet state is obtained, that is, $0.008 \mathrm{eV}(0.013 \mathrm{eV})$ above the triplet ground state for an STO MCSCF calculation with 12 active orbitals (for an cc-pVDZ MCSCF calculation with two active orbitals). Based in the experience provided for chains, at the beginning of this section, we guess that this small splitting should further reduce for more separate defects on a zigzag edge. On the other hand, the result obtained by a CR-CCSD(T) computation gives a relative large splitting $(0.35 \mathrm{eV})$, and what is more important it makes the singlet to be the ground state. This result shows the limitation of a computation that starts from a unique configuration when several molecular orbitals are degenerated. Notice that the RHF energy for the singlet is more than $4 \mathrm{eV}$ above the corresponding ROHF energy for the triplet. Consequently, coupled-cluster approximation should make for this difference when correlating the RHF state. In fact, a value of 0.026 for the $\mathrm{T} 1$ diagnostic points to a nonaccurate result. In conclusion, we get about one hundredth of $\mathrm{eV}$ as the splitting between singlet and triplet states, a value which confirms the weakness of spin correlation among these defects. As said before, our computational results point to more or less independent defects that will behave as isolated doublets.

An alternative way to show the subtle difference between triplet and singlet states is given by the difference between

TABLE II. Energy of the lower states of tetra-hydrogenated naphthacene shown in Fig. 4. Ab initio energies have been obtained using an MCSCF including $14 p_{z}$ active orbitals describing 14 electrons. The geometry is optimized at this level. Corrected energies that include MP2 additional correlation are given in parentheses. The last two columns show exact results for PPP model.

\begin{tabular}{llccc}
\hline \hline Spin multiplicity & ab initio energy (H.) & ab initio splitting (eV) & PPP energy (eV) & PPP splitting (eV) \\
\hline 1 & $-689.4232(-690.2684)$ & $0.40(0.34)$ & -135.98 & 0.23 \\
3 & $-689.4302(-690.2745)$ & $0.21(0.18)$ & -136.10 & 0.10 \\
5 & $-689.4378(-690.2810)$ & $0.0(0.0)$ & -136.21 & 0.0 \\
\hline \hline
\end{tabular}




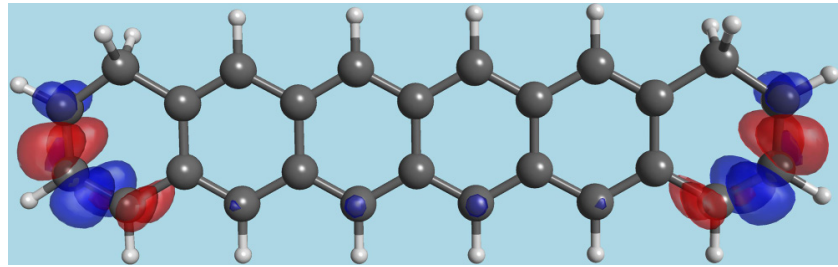

FIG. 5. (Color online) Di-hydrogenated hexacene representation. The charge difference between triplet ground state and singlet excited state is given at a contour value of $0.0001 \AA^{-3}$.

total electronic charges of both states. Figure 5 shows that difference; it is shallow and mainly localized near the bonds between $\mathrm{C}$ atoms that are close to the molecule ends.

\section{B. Large di-hydrogenated $[n]$ phenacenes}

This section constitutes the more important part of our work since defects at the ends of PAH's molecules having the shape of phenanthrene and picene are studied as a function of system size to show that their interaction vanishes for large enough separation. Although we have conducted tests for cases in which Lieb's theorem (or Hund's rule) predicts a ground state of spin multiplicity equal to three, here we will only present results obtained for clusters designed to have a singlet ground state, i.e., results obtained for balanced portions of graphene lattices. Figure 6 shows the largest di-hydrogenated $[n]$ phenacene that we have studied $\left(\mathrm{H}_{2}\right.$ [13]phenacene). It contains $54 \mathrm{C}$ atoms that are all coordinated with three atoms except at the edges where additional $\mathrm{H}$ atoms lead to fourfold coordination. Consequently, two $\pi$ orbitals are subtracted from the band forcing the appearance of two unpaired electrons on molecular orbitals with an energy close to 0 . This result can be straightforwardly obtained by means of a Hückel calculation and it is corroborated by elaborated $a b$ initio computation. In principle, two paired electrons can occupy the lowest molecular level but since LUMO-HOMO separation reduces rapidly for increasing cluster sizes, the question arises of whether we will end with an orbital degeneration and a triplet ground state. Actually, a closer look to any mean-field solution shows that hydrogenation produces bona fide localized states near the fourfold $\mathrm{C}$ atoms, no matter its sublattice. Assuming that Coulomb repulsion disfavors double occupation of any of them, two electrons should singly occupy both defect states producing either a singlet or a triplet ground state. If Lieb's theorem were still valid for the complex many-body Hamiltonian, the resulting ground state would be singlet for balanced molecules but triplet for unbalanced cases. It

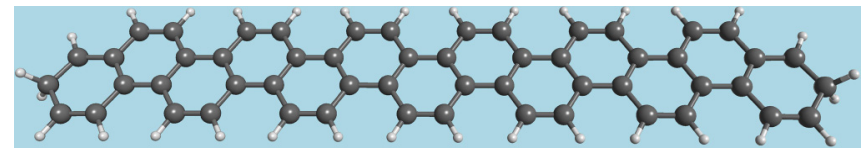

FIG. 6. (Color online) The largest di-hydrogenated $[n]$ phenacene $\left(n=13, \mathrm{C}_{54} \mathrm{H}_{32}\right)$ studied in our work.

would be quite embarrassing if some unknown long-distance interaction would pair localized distant electrons only if they were residing on different sublattices. The most simple remedy to avoid this unwanted scenario is a singlet-triplet splitting that vanishes for distant defects. Our numerical results prove that this is certainly the case, and consequently both localized electronic states occupied by one electron of any spin polarization define radical centers. Since they do not interact or interact weakly at medium distances, long di-hydrogenated phenacenes provide biradical examples. ${ }^{13}$

Lanczos results obtained for the PPP model on dihydrogenated $[n]$ phenacenes are shown in Fig. 7. Energies of the lowest singlet and triplet many-body states are plotted as a function of the inverse number of configurations $\left(N^{-1}\right)$ for systems including six to thirteen benzene rings (data are represented by circles). These results have been fitted by a law of the form:

$$
E=a_{0}+a_{1} / N^{1 / 4}+a_{2} / N^{1 / 2}+a_{3} / N,
$$

with very good results (relative differences between fitted and real values are about $10^{-6}$ ). In this way, extrapolated values can be obtained for the complete Hilbert space, that is, for the very large number of configurations limit $(1 / N \approx 0)$. Extrapolated values are represented by triangles, up triangle for the singlet and down for the triplet. Energies are always referred to the extrapolated ground state of the system, which is most probably a singlet. In our opinion, both the PPP model and the numerical approximate solution work very well although the final singlet-triplet splitting is a bit beyond the true accuracy of the procedure. Nevertheless, notice that although the correlation energy between $\pi$ electrons amounts several $\mathrm{eV}$ for both states, the final difference is well below $0.1 \mathrm{eV}$ independently of the fine details. Later, we will compare model results with singlet-triplet splittings obtained by ab initio computations.

Singlet and triplet states of di-hydrogenated $[n]$ phenacenes have also be obtained by means of standard multiconfiguration quantum-chemistry methods. It is possible to get a first insight into the level structure of these modified molecules using any semiempirical wave-function calculation, like MNDO or RM1 that are included in GAMESS package. ${ }^{30}$ The main characteristic

TABLE III. Energy of the lower states of di-hydrogenated hexacene shown in Fig. 5. Ab initio energies have been obtained using an STO basis in a MCSCF calculation describing 12 electrons within $12 p_{z}$ active molecular orbitals. The geometry is optimized at this level. Corrected energies that include MP2 additional correlation are given in parentheses. The third column gives the CR-CCSD(T) energies: first, the reference state, then, the correlated energy in parentheses. Finally, the fourth column provides MCSCF results obtained with a larger basis (cc-pVDZ) but only two electrons in two active molecular orbitals.

\begin{tabular}{llll}
\hline \hline Spin multiplicity & STO MCSCF energy (H.) & Coupled Cluster energy (H.) & cc-pVDZ MCSCF energy (H.) \\
\hline 1 & $-992.770751(-994.188179)$ & $-992.449825(-994.458893)$ & $-995.064021(-998.475755)$ \\
3 & $-992.770825(-994.188477)$ & $-992.599264(-994.446146)$ & $-995.064177(-998.476242)$ \\
\hline \hline
\end{tabular}




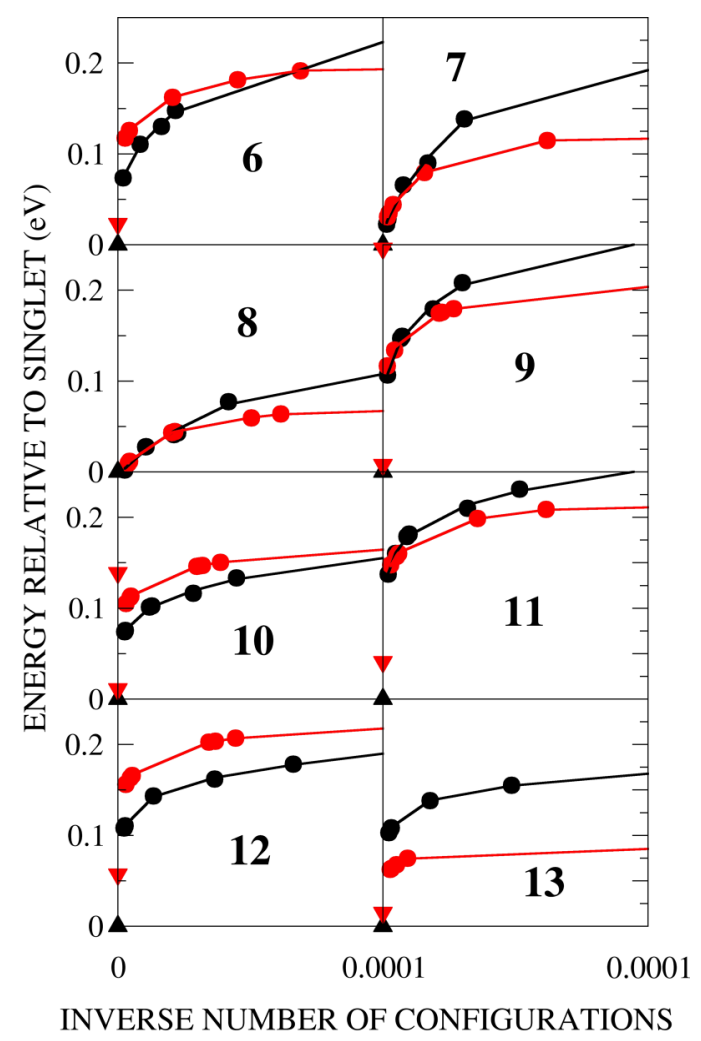

FIG. 7. (Color online) Lanczos results for the PPP model on di-hydrogenated $[n]$ phenacenes, for $n$ from six to thirteen (the one represented in Fig. 6). Energies are given as a function of the inverse of the number of many-body configurations used in the calculation. Only results for more than $10^{4}$ configurations are shown although the starting point is always one configuration, the one corresponding to the mean-field solution. The maximum number approximates typically one million in all graphs. Black circles give the energy of the lowest singlet, red circles correspond to the lowest triplet, and lines are fits. Energies refer to the singlet ground state that is obtained by extrapolation and represented by a black triangle up. The corresponding triplet extrapolated state is shown by a red triangle down (invading the lower panel for seven and eight rings cases).

is the appearance of two new levels between occupied bonding and unoccupied nonbonding molecular orbitals. They are well separated from both groups and correspond to bonding and nonbonding combinations of two states that are localized at the molecule ends. Actually, their amplitude decays exponentially towards the molecule center. We will show that the filling of these levels with electrons of any spin barely influences the final interacting energy of large enough systems. As a zero-order approximation, we start considering only spin configurations obtained for two electrons on these two molecular orbitals. Results for the difference between the lowest singlet (the ground state) and the lowest triplet are compiled in Fig. 8 as black circles. A simple STO-6G basis has been used and MCSCF energies have been corrected by second-order perturbation theory. Notice the use of a logarithmic vertical scale to emphasize the exponential decay for systems including five or more benzene rings. In order to improve the treatment of correlation, all possible fillings of the twelve MO-s around half-filling, i.e., the five upper bonding MO-s and the five lower non-bonding MO-s have been considered. The corresponding

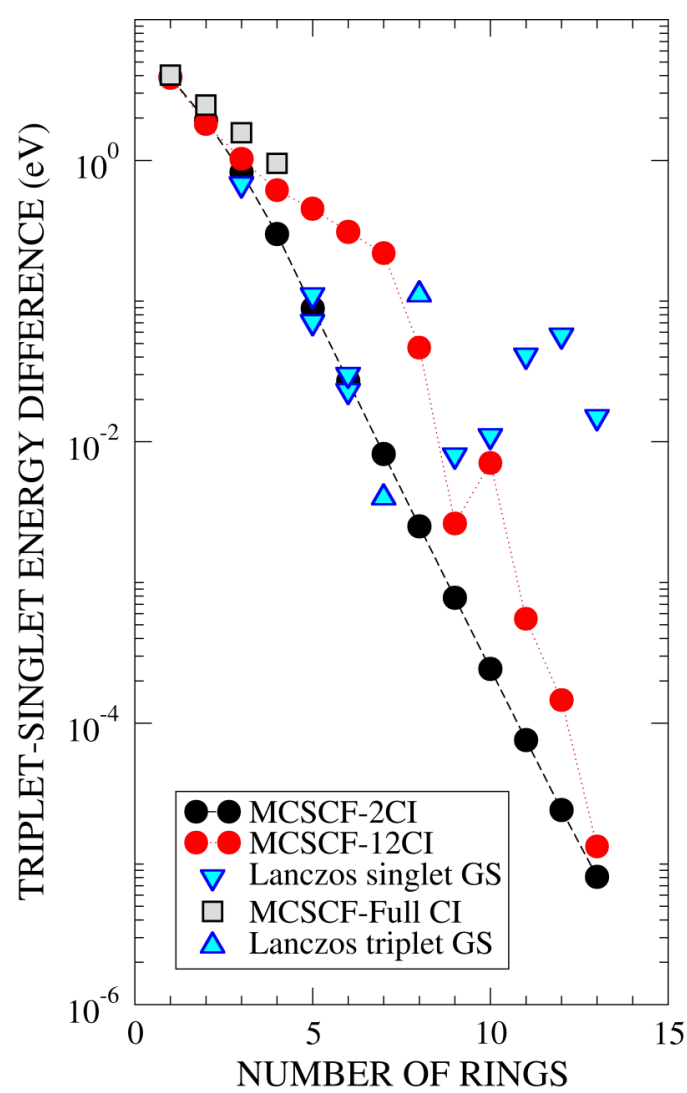

FIG. 8. (Color online) Triplet-singlet energy differences obtained for di-hydrogenated $[n]$ phenacenes of increasing size by means of several approaches. MCSCF results for the smallest number of configurations (black circles), for a large number of configurations (red circles), and exact results including all possible configurations for $\pi$ electrons (gray squares) are given for $a b$ initio computations and compared with results obtained by our Lanczos procedure for solving PPP model (down triangles for positive differences and up triangles for negative ones).

singlet-triplet splittings are given by the red circles. As before, they correspond to an STO-6G basis and have been corrected up to second order. The first visual impact is discouraging since the simple regularity of the first set of results has been substituted by an apparently chaotic behavior. Fortunately, there is an explanation for these results. Although the number of considered configurations has risen to several millions, this is still a small part of the total space for large systems. Moreover, the choice of configurations based only on the Hartree-Fock mean-field result is not necessarily the best one. As a consequence, a systematic increase of the triplet-singlet energy difference is obtained for the smaller systems while the increase for larger systems is neither monotonic nor proportional to the original difference. In order to have a robust result that supports a correct conclusion, we have calculated the whole correlation energy between $\pi$ electrons for the smaller systems. For the systems formed by one, two, three (di-hydrogenated phenanthrene), and four rings, all the possible configurations have been included and the geometry is optimized separately for singlet and triplet states. The resulting splittings are shown as gray squares and follow a perfect straight line with a slope sensibly smaller than the one followed 
TABLE IV. Total energies of [4]phenacene [two less rings than [6]phenacene shown in Fig. 2(c)] given by different approaches. RHF energy is used as energy origin. The fourth column includes correlation energy given by second order perturbation theory.

\begin{tabular}{lccc}
\hline \hline [4]phenacene & $\mathrm{S}(\mathrm{S}+1)$ & Mean field $(\mathrm{eV})$ & MP2 corrected $(\mathrm{eV})$ \\
\hline RHF & 0 & 0 & -31.23 \\
ROHF & 2 & -2.35 & -30.70 \\
UHF & 3.73 & -2.43 & -24.59 \\
CI & 0 & -10.72 & -34.40 \\
CI & 2 & -10.27 & -33.83 \\
\hline \hline
\end{tabular}

by the former approximations. We conclude that although interaction effects significantly amplify singlet-triplet splitting (and consequently, singlet stability), still the energy difference decreases exponentially as a function of system size.

A connection between the model and ab initio calculations can be established including Lanczos results in Fig. 8. The calculated model splittings are shown as triangles, down for positive triplet minus singlet energies but up for negative differences. It seems that model results follow the behavior of black circles until either the limited number of considered configurations or the uncertainty of extrapolated values fails to give a systematic behavior. In any case, even the results for larger systems fall below the imaginary straight line defined by the exact results given by gray squares.

Let us end this subsection giving total energies for [4]phenacene as obtained using different approximations (see Table IV). UHF gives the best monodeterminantal approximation, which differs more that $8 \mathrm{eV}$ from the singlet energy obtained by full CI. Differences are much smaller in the fourth column where energies include correlation energy up to second order. Notice that in this case, the correlations among all valence electrons are taken into account while the full CI correlation is limited to $\pi$ electrons.

\section{Hexa-hydrogenated coronene $\left(\mathrm{C}_{24} \mathrm{H}_{12}\right)$ and supercoronene $\left(\mathrm{C}_{54} \mathrm{H}_{18}\right)$}

Coronene and supercoronene (dodeca-benzo-coronene) are large PAH's molecules showing a regular hexagonal shape. They provide a convenient model for graphene flakes, probably not so regular. Some years ago, we showed that wisely designed hydrogenation of coronene produces molecules of large spin multiplicity. ${ }^{4}$ Here, we are interested not only in ground states but also in excited states, or more precisely in the difference between ground state and the first spin excited state. Therefore we will use a multireference starting point for a better description of states showing different spin multiplicities. Figure 9 shows the predicted "magnetic" molecule after complete saturation of six $\mathrm{C}$ atoms at the coronene contour while Fig. 10 shows a similar hexa-hydrogenated supercoronene designed to give an heptuplet ground state. A Hückel calculation gives six molecular orbital energies at zero separating bonding from antibonding states. We have performed MCSCF CI calculation based on these orbitals using either a very precise basis $\left(\mathrm{PC}^{23}\right)$ or the former simpler STO basis. ${ }^{21}$ Additionally, extra correlation energy has been

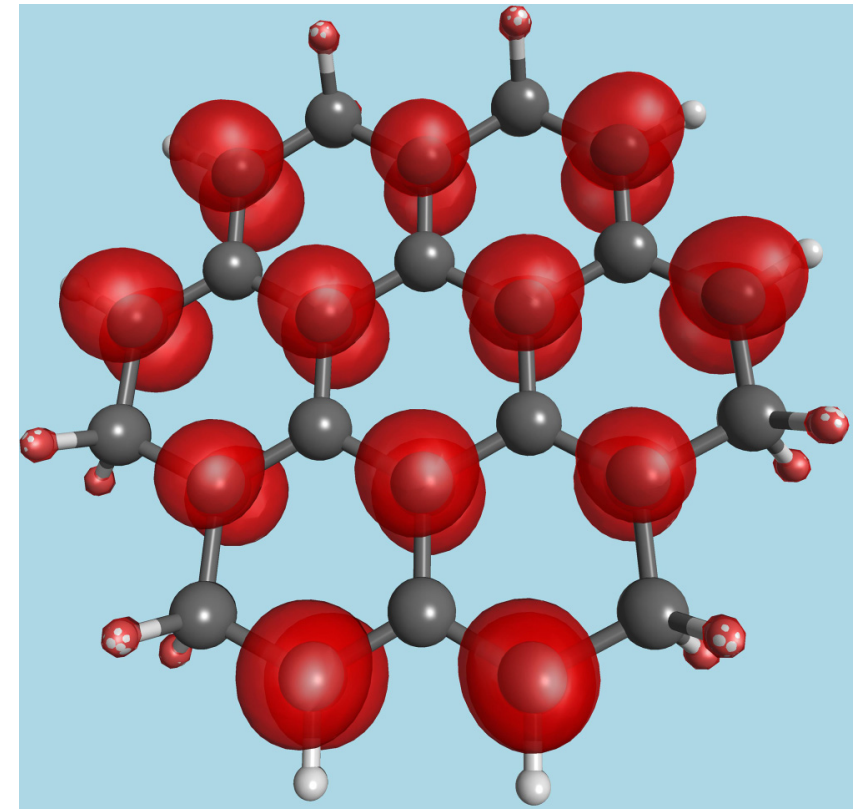

FIG. 9. (Color online) Hexa-hydrogenated coronene representation. Total spin density at a contour value of $0.007 \AA^{-3}$ is given for the ROHF approximation just to show how it resides on only one sublattice.

calculated by second-order perturbation theory for the STO basis set. The results are compiled in Table V showing that independent of the kind of calculation, all multiplets are within less than an electron-volt of energy. This result improves our previous computation where splittings amount several electron-volts. Figure 9 shows the spin density of an ROHF calculation for the heptuplet. The spin density is completely localized on the sublattice having more sites. This picture is a bit misleading since a correlated heptuplet for $S_{z}=0$ can also be obtained and then the numbers of up- and down-spin electrons are equal. Unfortunately, MACMOLPLT is still unable

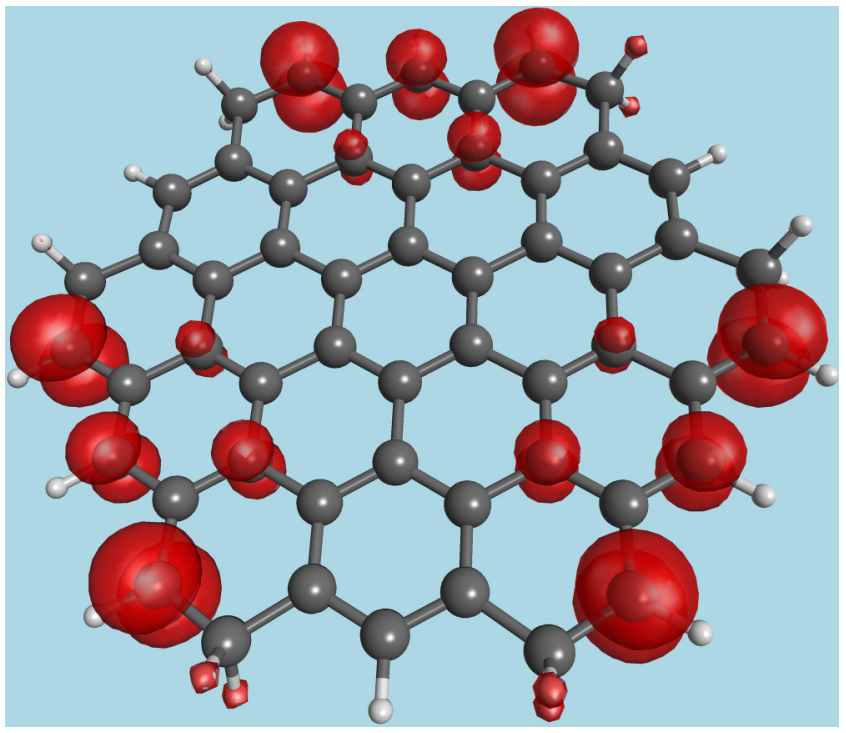

FIG. 10. (Color online) Hexa-hydrogenated supercoronene representation. Total spin density at a contour value of $0.005 \AA^{-3}$ is given to show how it separates in three disjoint molecule regions. 
TABLE V. Energy of the lower states of hexa-hydrogenated coronene. A large basis has been used for the MCSCF calculation (PC2) but CI has been restricted to the six $\pi$ orbitals that are parallel for the heptuplet state. The second column gives energies relative to the ground state (results for the smaller STO basis are given in parentheses for comparison). The last column gives STO splitting energies after including second-order corrections.

\begin{tabular}{lccc}
\hline \hline $2 S+1$ & $\begin{array}{c}\text { ab initio } \\
\text { energy (H.) }\end{array}$ & $\begin{array}{c}\text { ab initio } \\
\text { splitting (eV) }\end{array}$ & $\begin{array}{c}\text { MP2 ab initio } \\
\text { splitting (eV) }\end{array}$ \\
\hline 1 & -919.3849 & $0.72(0.59)$ & 0.84 \\
3 & -919.3884 & $0.62(0.49)$ & 0.68 \\
5 & -919.3978 & $0.37(0.23)$ & 0.33 \\
7 & -919.4113 & $0.0(0.0)$ & 0.0 \\
\hline
\end{tabular}

to represent the spin density of a correlated MCSCF wave function. In any case, the calculated multiplet separations are not so surprising for defect states that overlap considerably as it happens in this molecule.

Hexa-hydrogenation of supercoronene follows the usual scheme to get a ground state of large spin multiplicity. Figure 10 gives some details. Since six C atoms of the same sublattice become deactivated, a ground state of spin $S=3$ is expected both by Hund's rule after diagonalization of Hückel model and Lieb's theorem. But the interesting novelty appears after doing ROHF for a better description of the heptuplet. As Fig. 10 shows, the spin density associated to the unpaired electrons is now localized in three different regions. This fact implies smaller differences for the energy of different spin configurations as we have repeatedly found in previous sections. Table VI gives our computational results. Splittings among multiplets have been cut off by a factor of ten relative to coronene splittings. We argue that this behavior is similar to the one found for quasilinear systems and di-hydrogenated $[n]$ phenacenes. Consequently, we expect a polyradical behavior for distant defects independently of their distribution between sublattices. Again, Lieb's theorem works for real PAH molecules but spin excitations are so close that they will destabilize the well-defined spin of the ground state at finite temperatures. In such situation, the finding of long-range magnetic correlations with origin in local defects does not seem plausible.
TABLE VI. Energy of the lower states of hexa-hydrogenated supercoronene relative to the ground state, which is sevenfold spin degenerated. Values have been obtained for an STO basis and 12 active orbitals occupied by 12 electrons in an MCSCF approximation. The last column includes the correlation energy given by second-order perturbation theory.

\begin{tabular}{lcc}
\hline \hline $2 S+1$ & ab initio splitting $(\mathrm{eV})$ & MP2 ab initio splitting (eV) \\
\hline 1 & 0.013 & 0.061 \\
3 & 0.011 & 0.058 \\
5 & 0.007 & 0.034 \\
7 & 0.0 & 0.0 \\
\hline \hline
\end{tabular}

\section{CONCLUDING REMARKS}

All the systems we have calculated show that the energies of the lowest singlet and the lowest triplet states become closer as the distance between the defects giving rise to new states within the original HOMO-LUMO gap increases. This feature could have been expected based on the localized nature of defect states. Nevertheless, many-body interactions deeply influence the actual singlet-triplet splitting energy and could have eventually produced long-range spin interactions. Our most systematic computations for long di-hydrogenated $[n]$ phenacenes prove that although correlation greatly increases the stability of singlet relative to triplet, this energy difference is of little relevance due to its exponential decay as a function of the length of the molecule. We conclude that this kind of defects are populated by unpaired electrons that should be described as localized radical centers. All systems should exhibit a polyradical character, and any ordered spin structure should be very improbable for defects separated well enough. Finally, we suggest that defect centers can interact with currents of electrons via spin exchange at a small energetic cost.

\section{ACKNOWLEDGMENTS}

Financial support by the Spanish MICINN (MAT201126534, CTQ2007-65218, CSD2007-6, FIS2009-08744, and CTQ2011-24165) and the Universidad de Alicante is gratefully acknowledged. We also acknowledge support from the DGUI of the Comunidad de Madrid under the R\&D Program of activities MODELICO-CM/S2009ESP-1691.

\footnotetext{
*jav@icmm.csic.es

${ }^{1}$ K. Kusakabe and M. Maruyama, Phys. Rev. B 67, 092406 (2003).

${ }^{2}$ O. V. Yazyev, Rep. Prog. Phys. 73, 056501 (2010).

${ }^{3}$ R. R. Nair, M. Sepioni, I.-Ling Tsai, O. Lehtinen, J. Keinonen, A. V. Krasheninnikov, T. Thomson, A. K. Geim, and I. V. Grigorieva, Nat. Phys. 8, 199 (2012).

${ }^{4}$ J. A. Vergés, G. Chiappe, E. Louis, L. Pastor-Abia, and

E. San-Fabián, Phys. Rev. B 79, 094403 (2009).

${ }^{5}$ E. H. Lieb, Phys. Rev. Lett. 62, 1201 (1989).

${ }^{6}$ J. Fernández-Rossier and J. J. Palacios, Phys. Rev. Lett. 99, 177204 (2007).
}

${ }^{7}$ J. A. Vergés, E. San-Fabián, L. Pastor-Abia, G. Chiappe, and E. Louis, Phys. Stat. Solidi. C 6, 2139 (2009).

${ }^{8}$ E. San-Fabián, A. Guijarro, J. A. Vergés, G. Chiappe, and E. Louis, Eur. Phys. J. B 81, 253 (2011).

${ }^{9}$ R. Pariser and R. G. Parr, J. Chem. Phys. 21, 466 (1953).

${ }^{10}$ J. A. Pople, Trans. Faraday Soc. 49, 1375 (1953).

${ }^{11}$ J. A. Vergés, E. San-Fabián, G. Chiappe, and E. Louis, Phys. Rev. B 81, 085120 (2010).

${ }^{12}$ X. Zhang, O. V. Yazyev, J. Feng, L. Xie, C. Tao, Y.-C. Chen, L. Jiao, Z. Pedramrazi, A. Zetti, S. G. Louie, H. Dai, and M. F. Crommie, ACS Nano 7, 198 (2013). 
${ }^{13}$ Compendium of Chemical Terminology, 2nd ed. (the "Gold Book") edited by A. D. McNaught and A. Wilkinson (Blackwell Scientific Publications, Oxford, 1997). XML on-line corrected version: http://goldbook.iupac.org (2006) created by M. Nic, J. Jirat, and B. Kosata; updates compiled by A. Jenkins, doi: $10.1351 /$ goldbook.

${ }^{14}$ E. San-Fabián, J. A. Vergés, G. Chiappe, and E. Louis (unpublished).

${ }^{15}$ M. W. Schmidt, K. K. Baldridge, J. A. Boatz, S. T. Elbert, M. S. Gordon, J. H. Jensen, S. Koseki, N. Matsunaga, K. A. Nguyen, S. J. Su, T. L. Windus, M. Dupuis, and J. A. Montgomery, J. Comput. Chem. 14, 1347 (1993).

${ }^{16} \mathrm{M}$. S. Gordon and M. W. Schmidt, in Theory and applications of Computational Chemistry, the First Forty Years, edited by C. E. Dykstra, G. Frenking, K. S. Kim, and G. E. Scuseria (Elsevier, Amsterdam, 2005), p. 1167.

${ }^{17}$ C. Møller and M. S. Plesset, Phys. Rev. 46, 618 (1934).

${ }^{18}$ H. Nakano, J. Chem. Phys. 99, 7983 (1993).

${ }^{19}$ J. A. Vergés, P. L. de Andres, E. San-Fabián, G. Chiappe, E. Louis, and A. Guijarro, Phys. Rev. B 85, 165102 (2012).
${ }^{20} \mathrm{~A}$ very useful interactive program that performs Java calculations for Hückel model can be found at http://www.chem. ucalgary.ca/SHMO/

${ }^{21}$ W. J. Hehre, R. F. Stewart, and J. A. Pople, J. Chem. Phys. 51, 2657 (1969).

${ }^{22}$ T. H. Dunning Jr., J. Chem. Phys. 90, 1007 (1989).

${ }^{23}$ F. Jensen, J. Chem. Phys. 115, 9113 (2001).

${ }^{24}$ MACMOLPLT package has been employed to represent the calculated clusters. It is described by B. M. Bode and M. S. Gordon, J. Mol. Graphics Mod. 16, 133 (1998).

${ }^{25}$ D. A. Papaconstantopoulos, Handbook of the Band Structure of Elemental Solids (Plenum Press, New York, 1986).

${ }^{26}$ K. Ohno, Theor. Chim. Acta 2, 219 (1964).

${ }^{27}$ R. J. Buenker and S. D. Peyerimhoff, Theor. Chim. Acta 35, 33 (1974).

${ }^{28}$ W. L. Wang, O. V. Yazyev, S. Meng, and E. Kaxiras, Phys. Rev. Lett. 102, 157201 (2009).

${ }^{29}$ S. Fajtlowicz, P. E. John, and H. Sachs, Croat. Chem. Acta 78, 195 (2005).

${ }^{30}$ The use of the Java interactive program (see Ref. 20) becomes unpractical for large systems. 